EPJ Web of Conferences 59, 13007 (2013)

DOI: $10.1051 /$ epjconf/20135913007

(C) Owned by the authors, published by EDP Sciences, 2013

\title{
High-resolution K-shell spectra from laser excited molybdenum plasmas
}

\author{
C.I. Szabo ${ }^{1}$, P. Indelicato ${ }^{1}$, L.T. Hudson ${ }^{2}$, J.F. Seely ${ }^{3}$ and T. Ma ${ }^{4}$ \\ ${ }^{1}$ Laboratoire Kastler Brossel, ENS, CNRS, UPMC, Paris, France \\ ${ }^{2}$ National Institute of Standards and Technology, Gaithersburg, MD, USA \\ 3 U.S. Naval Research Laboratory, Washington DC, USA \\ ${ }^{4}$ Lawrence Livermore National Laboratory, Livermore, CA, USA
}

\begin{abstract}
X-ray spectra from Molybdenum plasmas were recorded by a Cauchois-type cylindrically bent Transmission Crystal Spectrometer (TCS). The absolutely calibrated spectrometer provides an unprecedented resolution of inner shell transitions ( $\mathrm{K}$ x-ray radiation). This tool allows us to resolve individual lines from different charge states existing inside the laser-produced plasma. The inner shell transitions from highly charged Molybdenum shown in this report have never been resolved before in such detail in a laser-produced plasma.
\end{abstract}

\section{INTRODUCTION}

The exploration of atomic processes by observing inner shell transitions is a powerful tool in determining plasma properties e.g. charge-state distributions, electron and ion temperatures, densities and opacities. The conditions observable in laser-produced plasmas in the laboratory can also relate to astrophysical observations. The observation of atomic line spectra at high-resolution originating from high-energydensity plasmas gives unique insight into the validity of atomic physics codes under these extreme conditions and also can provide a way to observe transitions in highly charged ions that are difficult if not impossible to observe in the laboratory in other ways.

In many high-energy laser experiments an x-ray source (usually point like) is used offset from the main target, to excite or backlight processes in the primary high-density plasma. To create this $\mathrm{x}$-ray source, some high-energy laser beams are focused on a solid foil target. With recent increases in energy density achieved with laser irradiation of solids, the energy of the x-ray sources backlighting in point projection radiography or used for Thomson scattering has shifted to higher x-ray energies, permitting the study of the emitted $x$ rays of laser-excited mid-Z elements [1].

\section{EXPERIMENTAL SETUP}

The transmission crystal spectrometer (TCS) is a standard diagnostic at the Laboratory for Laser Energetics (LLE) at the University of Rochester and facilitates the recording of high-resolution x-ray spectra of K shell transitions in heavy elements [1-3]. TCS was designed and built by a joint team from the National Institute of Standards and Technology (NIST) and the U.S. Naval Research Laboratory (NRL) for use at LULI (Laboratoire pour l'Utilisation des Lasers Intenses), Paris. It is currently on long-term loan to LLE and is qualified for use in TIMs (ten inch manipulators) on the EP and OMEGA target chambers.

This is an Open Access article distributed under the terms of the Creative Commons Attribution License 2.0, which permits unrestricted use, distribution, and reproduction in any medium, provided the original work is properly cited. 


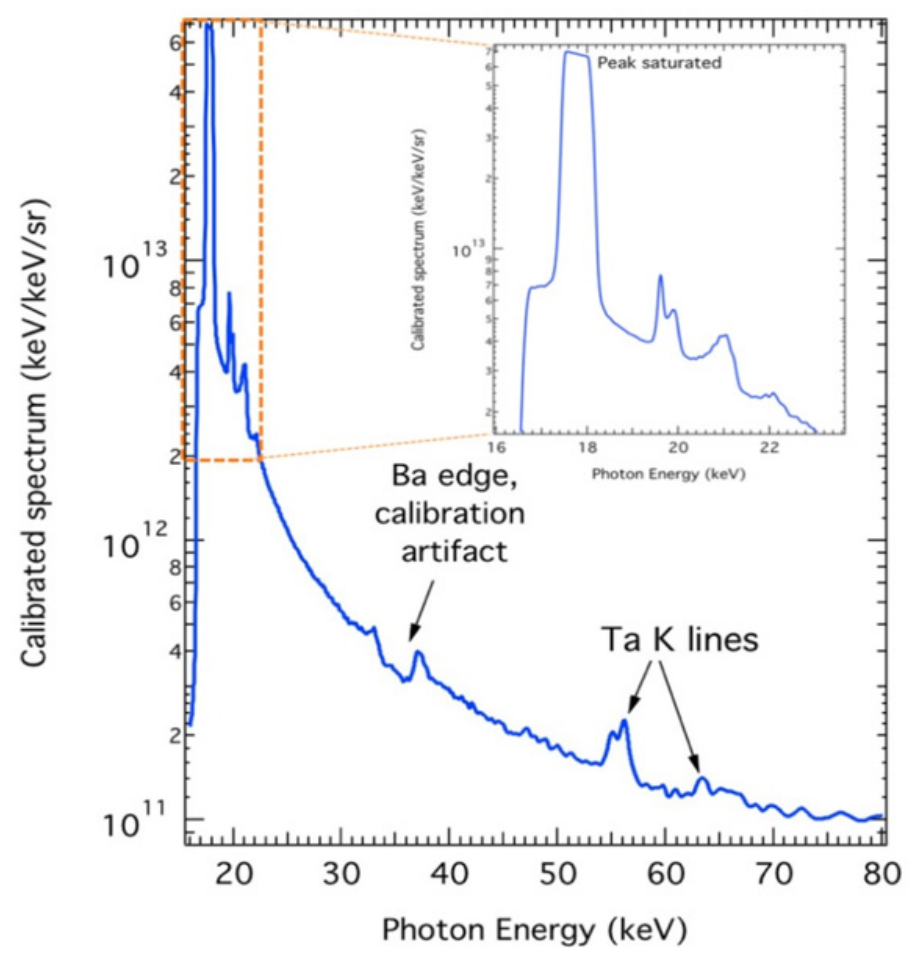

Figure 1. Calibrated TCS spectrum from IP position A. The insert shows a zoom of the marked region.

The TCS consists of a cylindrically bent crystal in the Cauchois geometry, a crossover slit, and four image plate detector packs in four positions behind the wavelength-dispersing optic. The crystal is quartz (10-11) with a $2 \mathrm{~d}=0.668 \mathrm{~nm}$ lattice spacing, a $0.29 \mathrm{~mm}$ thickness, and $254 \mathrm{~mm}$ bending radius. The crystal is positioned at $600 \mathrm{~mm}$ distance from the target, the crystal to slit distance is $105 \mathrm{~mm}$. The nosecone is equipped with a removable pointer and a debris shield [4].

The TCS was used to record X-ray spectra from laser-induced fluorescence of Mo foils used as the $\mathrm{X}$-ray source for a high-energy Thomson scattering experiment at the OMEGA laser facility. TCS was directly pointed to the source of $x$ rays, collecting data on all four image plate (IP) positions; the first two IP's had a bandwidth coverage that included the Mo K x-ray lines. In the Omega shot \#59891 analyzed in this report 16 backlighter beams $(\sim 500 \mathrm{~J})$ with a total energy of $\sim 8 \mathrm{~kJ}$ were focused to a $12 \mu \mathrm{m}$ thick Mo foil that was serving as the x-ray source in the Thomson scattering experiment.

\section{SPECTRA IN HIGH RESOLUTION}

Figure 1 shows the full Mo spectrum registered by TCS on the Rowland circle, IP position A. This figure shows the spectrum on an absolute intensity scale that is based on a calibration of the crystal reflectivity [5] and the image plate efficiency [6]. One can observe the $\mathrm{K}$ shell lines of neutral and highly charged molybdenum in the $17 \mathrm{keV}$ to $22 \mathrm{keV}$ energy range. At around $56 \mathrm{keV}$ x-ray energy the Tantalum $\mathrm{K} \alpha$ lines can be observed with a few orders of magnitude lower intensity. These lines originate from the target assembly that contained Ta in the structure and indicate that in these OMEGA shots, sufficiently high energy electrons are created that can excite these x-ray transitions in the target material. For a comparison of the spectral resolution with the image plates placed on the Rowland circle 
IFSA 2011

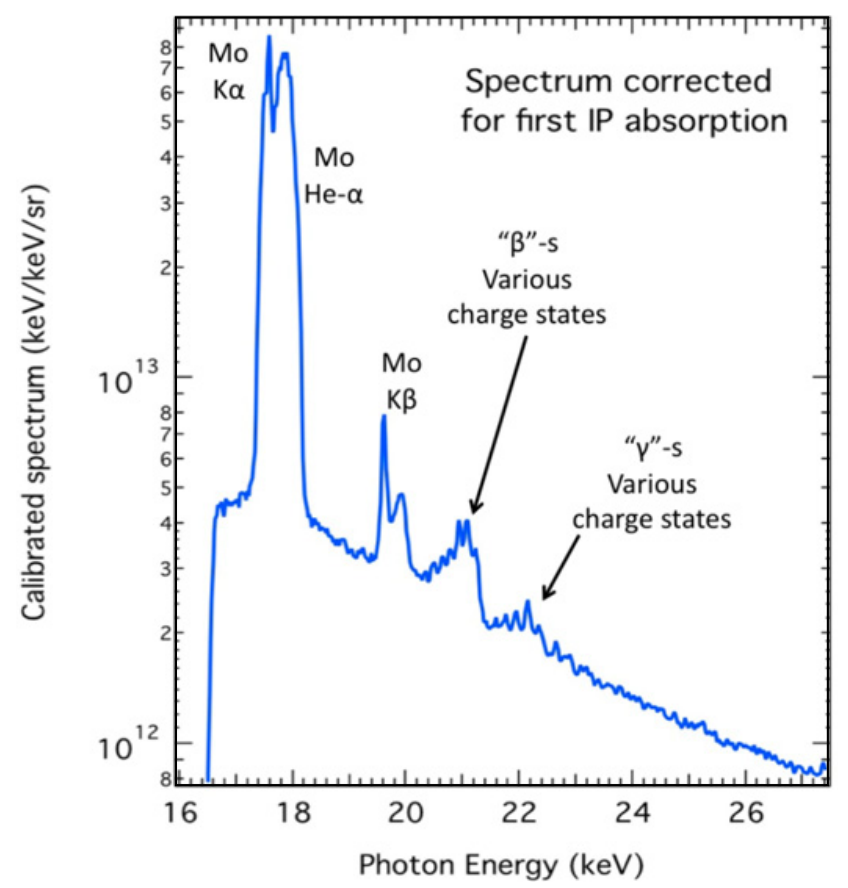

Figure 2. Calibrated TCS spectrum from IP position B. The spectrum has been corrected for the absorption of the image plate in IP position A.

(IP position A) and $200 \mathrm{~mm}$ off the Rowland circle (IP position B) one can observe the spectrum inset in Figure 1 with increased resolving power in Figure 2.

The calibrated scale on Figure 2 of the spectrum recorded on IP B was normalized to the intensities observed on IP A. As IP A is in front of IP B the absorption occurring in the first image plate was accounted for.

The spectrum on Figure 2 demonstrates the enhancement of instrumental resolving power of TCS when the detecting media is moved farther away from the crystal beyond the Rowland circle and the X-ray source is sufficiently point-like [7] such that source-size line broadening is small and the resolving power is dominated by the intrinsic resolution of the detection medium. A detailed study of the resolution of the conventionally used image plate detectors is given in reference [8]. The spectral resolving power at position $\mathrm{B}$ is about twice that measured in position $\mathrm{A}$.

\section{FLYCHK SIMULATIONS}

The Mo spectrum containing lines from several different charge states was simulated using the atomic physics code FLYCHK [9] with different electron temperatures as the input along with an electron density of $8 \times 10^{21} \mathrm{~cm}^{-3}$ without taking opacity into consideration. The results of these simulations are shown in Figure 3. It was found that a large thermal to hot electron ratio provides the best fit to the experimental data. The closest fit to the spectral x-ray continuum is marked with the thick line and represents the emissions from a plasma with a $4.5 \mathrm{keV}$ thermal electron temperature and $30 \mathrm{keV}$ hot electron temperature. Modeling the relative intensities of the observed characteristic lines proved difficult. This might be due to the fact that FLYCHK relies on a crude model based on hydrogenic wavefunctions only. Another reason could be that in the experimental spectrum line transitions due to neutral or close to neutral ions are observed in addition to lines from highly-charged states of Mo that is 


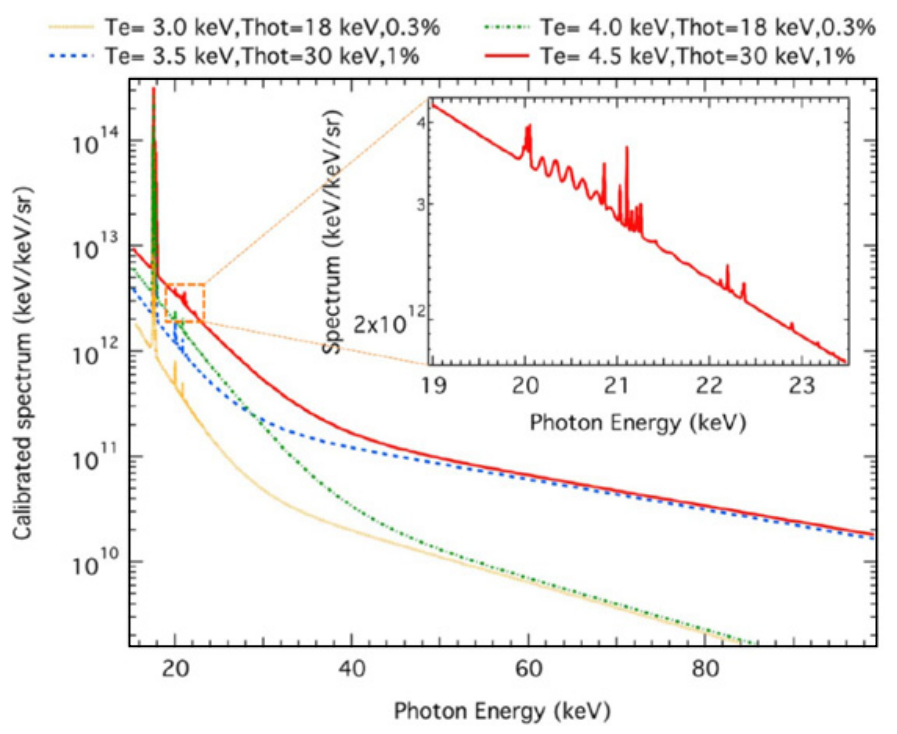

Figure 3. Result of various FLYCHK runs. The insert shows a zoom of the closest match for our spectra.

difficult to include in the FLYCHK simulation. Presumably the field of view of the backlighter source included a relatively cold plasma component, and the simulations should be extended to include such behavior. With further simulations that include opacity effects in the target and plasma, better agreement could be expected, as was the case with similar spectra and analysis described in [10].

\section{SUMMARY}

We have shown that TCS is capable of acquiring high-energy x-ray spectra in relatively high resolution, which is enhanced in the case of Molybdenum using the second image plate position to a resolving power above 500. In addition, due to a recent calibration of this instrument, we can present the spectral results on an absolute scale. The spectra can be modeled with the FLYCHK code to achieve good agreement with the observed X-ray continuum from Mo backlighter targets.

\section{References}

[1] J. F. Seely, C. I. Szabo, P. Audebert, E. Brambrink, E. Tabakhoff, G. E. Holland, L. T. Hudson, A. Henins, P. Indelicato, and A. Gumberidze, High Energy Density Phys. 5, 263 (2009)

[2] C. I. Szabo, P. Indelicato, A. Gumberidze, G. E. Holland, J. F. Seely, L. T. Hudson, A. Henins, P. Audebert, S. Bastiani-Ceccotti, E. Tabakhoff, and E. Brambrink, Eur. Phys. J. Special Topics 169, 243 (2009)

[3] J. F. Seely, C. I. Szabo, P. Audebert, E. Brambrink, E. Tabakhoff, and L. T. Hudson, Phys. Plasmas 17, $023102(2010)$

[4] J. F. Seely, C. I. Szabo, U. Feldman, L. T. Hudson, A. Henins, P. Audebert and E. Brambrink, Rev. Sci. Instrum. 81, 10E301 (2011)

[5] C. I. Szabo, U. Feldman, S. Seltzer, L. T. Hudson, M. O’Brien, H.-S. Park and J. F. Seely, Optics Lett. 36, 1335 (2011)

[6] B. R. Maddox, H. S. Park, B. A. Remington, N. Izumi, S. Chen, C. Chen, G. Kimminau, Z. Ali, M. J. Haugh, and Q. Ma, Rev. Sci. Instrum. 82, 023111 (2011)

[7] J. F. Seely, L. T. Hudson, G. E. Holland, and A. Henins, Appl. Opt. 47, 2767 (2008) 


\section{IFSA 2011}

[8] J. F. Seely, G. E. Holland, L. T. Hudson, and A. Henins, Appl. Opt. 47, 5753 (2008)

[9] H.-K. Chung, M.H. Chen, W.L. Morgan, Y. Ralchenko, R.W. Lee, High Energy Density Physics $\mathbf{1}, 3$ (2005)

[10] J. F. Seely, C.A. Back, C. Constantin, R.W. Lee, H.-K. Chung, L.T. Hudson, C.I. Szabo, A. Henins, G.E. Holland, R. Atkin and L. Marlin, Journal of Quantitative Spectroscopy \& Radiative Transfer 99, 572 (2006) 